\title{
X-ray emitting class I protostars in the Serpens dark cloud
}

\author{
T. Preibisch
}

\author{
Max-Planck-Institut für Radioastronomie, Auf dem Hügel 69, 53121 Bonn, Germany \\ e-mail: preib@mpifr-bonn.mpg.de
}

Received 4 June 2004 / Accepted 25 August 2004

\begin{abstract}
We analyze a set of three individual XMM-Newton X-ray observation of the Serpens dark cloud. In addition to the 45 sources already reported in the analysis of the first of these XMM-Newton observations by Preibisch (2003), the complete combined data set leads to the detection of X-ray emission from four of the 19 known class I protostars in the region. The set of three observations allows us to study the variability of the sources on timescales from minutes to several months. The lightcurves of two of the four X-ray detected class I protostars show evidence for significant variability; the data suggest at least four flare-like events on these objects. This relatively high level of variability in the X-ray emission from the class I protostars is in qualitative agreement with the result by Imanishi et al. (2001), who found that the class I protostars in the $\rho$ Ophiuchi dark cloud show a higher level of variability than that of more evolved class II and class III young stellar objects. This may support non-coronal X-ray emission mechanisms for class I protostars and is in agreement with the predictions of models that assume magnetic interactions between the protostar and its surrounding disk as a source of high-energy emission. We also find a strong variation (by a factor of $\sim 10$ ) in the X-ray luminosity of the class II object EC 74 between the three observations, which may be explained by a long duration flare or by rotational modulation. Finally, we find no evidence for X-ray emission from the five class 0 protostars in the region.
\end{abstract}

Key words. open clusters and associations: individual: stars: Serpens cloud - stars: formation - stars: coronae stars: pre-main sequence - X-rays: stars

\section{Introduction}

Young stellar objects (YSOs) can be classified into a (evolutionary) sequence of four main classes according to their infrared spectral energy distribution, which traces the amount of circumstellar material (see, e.g., Wilking et al. 2001). Class 0 protostars represent an extremely young stage in which most of the mass resides still in the circumstellar environment. Class I protostars have already accreted a considerable fraction of their final mass, but a major fraction of their energy output is still due to accretion. The canonically assumed ages of class I protostars are $\sim 10^{5}$ years. Class II objects (classical T Tauri stars with typical ages of up to a few $10^{6}$ year) are still surrounded by circumstellar material, but their accretion rates are already so low that the energetics of the system is dominated by the central star. Finally, the class III objects have fully accreted or dispersed their circumstellar material. As a further sub-division of this sequence, the so-called "flat spectrum" objects are in the transition between class I and class II.

Many YSOs in all evolutionary stages from class I protostars to young main-sequence stars show highly elevated levels of X-ray activity (for reviews see Feigelson \& Montmerle 1999; Favata \& Micela 2003). Since X-rays with energies above $\sim 1 \mathrm{keV}$ are much less affected by extinction than optical light, X-ray observations of star forming regions allow a deep look into dense molecular cloud cores towards the embedded YSOs. The high energy processes in YSOs are of great importance for our understanding of the star formation process. For example, the X-ray emission from a YSO photoionizes its circumstellar material and thus influences accretion as well as outflow processes, both of which are thought to be based on the coupling of ionized material with magnetic fields. As the protostellar X-ray emission is an important, perhaps even the dominant factor in determining the ionization structure of protoplanetry disks, it can have a strong impact on the formation of proto-planets (cf. Glassgold et al. 2000; Matsumura \& Pudritz 2003).

The origin of the strong X-ray emission in YSOs is still not fully understood. For class II and class III T Tauri stars, there is strong evidence that the X-ray emission comes from magnetically confined plasma in the stellar corona, and thus is the result of strongly enhanced, but in principle solar-like magnetic dynamo processes. The exact nature of the magnetic activity in T Tauri stars, however, is still not well known (see, e.g., Feigelson et al. 2003). The origin of the high-energy emission from class I protostars is even less well understood; for example, it is unclear whether these extremely young objects could already develop solar-like coronae. Their X-ray emission may well be dominated by non-coronal emission processes. Since the number of X-ray detected class I protostars is still relatively small, further observations are important to better characterize the $\mathrm{X}$-ray properties of this class of objects. 
Table 1. Observational parameters of the three exposure of the Serpens region discussed in this paper. We list the XMM Observation Identifier number, the satellite revolution, start and stop time, the exposure times for the PN and MOS cameras (without subtraction of high background interval times), the actual pointing direction in J2000 coordinates, and the roll angle for each of the observations.

\begin{tabular}{|c|c|c|c|c|c|c|c|c|}
\hline & $\begin{array}{l}\text { Obs } \\
\text { ident }\end{array}$ & Rev & $\begin{array}{c}\text { Date and Time } \\
{[\mathrm{UT}]}\end{array}$ & $\begin{array}{c}\text { Expo. PN } \\
{[\mathrm{ks}]}\end{array}$ & $\begin{array}{c}\text { Expo. MOS } \\
{[\mathrm{ks}]}\end{array}$ & $\begin{array}{c}\text { Pointing RA } \\
\text { [deg] }\end{array}$ & $\begin{array}{l}\text { Pointing Dec } \\
\text { [deg] }\end{array}$ & $\begin{array}{c}\text { Roll angle } \\
\text { [deg] }\end{array}$ \\
\hline Obs1 & 65820501 & 613 & 14.04.2003 14:09-17:36 & 10.1 & 12.4 & 277.4769 & 1.2324 & 66.70 \\
\hline Obs2 & 65820201 & 694 & 23.09.2003 01:51-08:37 & 18.0 & 20.3 & 277.5155 & 1.1935 & 270.00 \\
\hline Obs3 & 65720601 & 696 & 27.09.2003 01:35-08:13 & 18.0 & 19.7 & 2.775163 & 1.1940 & 268.37 \\
\hline
\end{tabular}

The Serpens dark cloud is a very active star forming region at a distance of $\sim 310 \mathrm{pc}$ (see Preibisch 2003 for a discussion of the distance). Most YSOs are highly obscured and only visible in the infrared. Infrared and mm observations of the cluster (Strom et al. 1976; Eiroa \& Casali 1992; Sogawa et al. 1997; Giovannetti et al. 1998; Kaas 1999; Hurt \& Barsony 1996; Testi \& Sargent 1998) have revealed more than 100 YSOs in all evolutionary states from protostellar condensations and class 0 protostars to class III T Tauri stars. A deep ISOCAM survey in the $6.7 \mu \mathrm{m}$ and $14.3 \mu \mathrm{m}$ bands allowed Kaas et al. (2004) to identify 66 YSOs with mid-infrared excesses; they classified 19 of these YSOs as class I protostars according to their near- to mid-infrared spectral energy distribution.

The first X-ray observation of the Serpens cloud, described in Preibisch (1998), was performed with the ROSAT HRI and revealed just seven X-ray sources within the 40' diameter fieldof-view. In Preibisch (2003) we recently reported the results of a new X-ray observation of the Serpens cloud with the $X M M-N e w t o n$ observatory; this observation was the first of the three data sets we analyze in this paper. XMM-Newton has not only a much larger collecting area than ROSAT, but is also sensitive over a much wider energy band, extending up to $\sim 15 \mathrm{keV}$, what makes it much better suited to study the strongly extincted and therefore very hard X-ray emission from highly obscured YSOs. In this first XMM-Newton observation, 45 individual $X$-ray sources were detected in the $\approx 30^{\prime}$ diameter field-of-view. Nine of these X-ray sources could be identified with class II+III T Tauri stars, two with flat-spectrum objects, one with a class I protostar (EC 53), and none with a class 0 protostar. This non-detection of most of the embedded very young stellar population (five class 0 objects and 19 class I protostars) is related to the relatively short exposure time of that observation. Meanwhile, two additional XMM-Newton observations of the Serpens cloud have been obtained. The combination of the previous and the new XMM-Newton data does not only increase the sensitivity, but also provides a very good opportunity to study the variability of the X-ray emitting objects on timescales from days to months.

\section{Observations and data analysis}

The observations discussed in this paper were obtained with the European Photon Imaging Cameras (EPIC) onboard of XMM-Newton. The EPIC system (see Jansen et al. 2001) consists of two identical EPIC-MOS cameras (Turner et al. 2001) and the EPIC-PN camera (Strüder et al. 2001). The cameras were operated with the medium optical blocking filter and in the Full-Frame mode. They provide a $\approx 30^{\prime}$ diameter field-ofview, energy coverage from $\approx 0.15-15 \mathrm{keV}$, and moderate energy resolution $(E / \Delta E \approx 20-50)$.

We use here a set of three individual XMM-Newton observations ${ }^{1}$. The details of these three exposures, which we will call Obs1, Obs2, and Obs3, are listed in Table 1.

Extraction of science products from the Observation Data Files followed standard procedures using the XMM-Newton Science Analysis System (SAS) version 5.4.1. To obtain an $\mathrm{X}$-ray image we merged all three data sets for all three detectors (PN, MOS1, and MOS2). In our analysis of the individual $\mathrm{X}$-ray sources we concentrated on the data of the PN camera, which yields the highest signal (countrates).

\section{New and strongly variable $X$-ray sources in the merged data set}

The central part of the merged XMM-Newton image is shown in Fig. 1. The by far brightest X-ray source is the "flat-spectrum" object EC 95, which is discussed in detail in Preibisch (2003). The comparison of the merged X-ray image to the image based on Obs1 only (as discussed in Preibisch 2003) revealed several new X-ray sources. Two new sources appear close to the very bright source EC 95; they can be identified with the class I protostars EC 80 and EC 89. Another new X-ray source is seen to the south-east of EC 95 and can be identified with the class I protostar PS 1 discussed by Hurth \& Barsony (1996). The X-ray properties of these sources will be discussed below in detail. With the exception of EC 53, which was already detected in the data from Obs1, the other 15 class I protostars in the region (see Kaas et al. 2004) remain undetected in the XMM-Newton image.

We also searched for X-ray emission at the position of the five known class 0 protostars in the Serpens cloud (Hurt \& Barsony 1996), but could not detect X-ray emission from any of these objects.

1 There exists another XMM-Newton observation (obtained on 12 April 2003), which however was strongly affected by a high, flaring radiation background and was therefore not used in our analysis; inspection of these data showed that none of the class I protostars discussed in this paper was detected in that observation. of the Serpens cloud that were obtained between April and September 2003. These three data sets were not affected by strong background variability, and their full exposure times could be use for scientific analysis. 


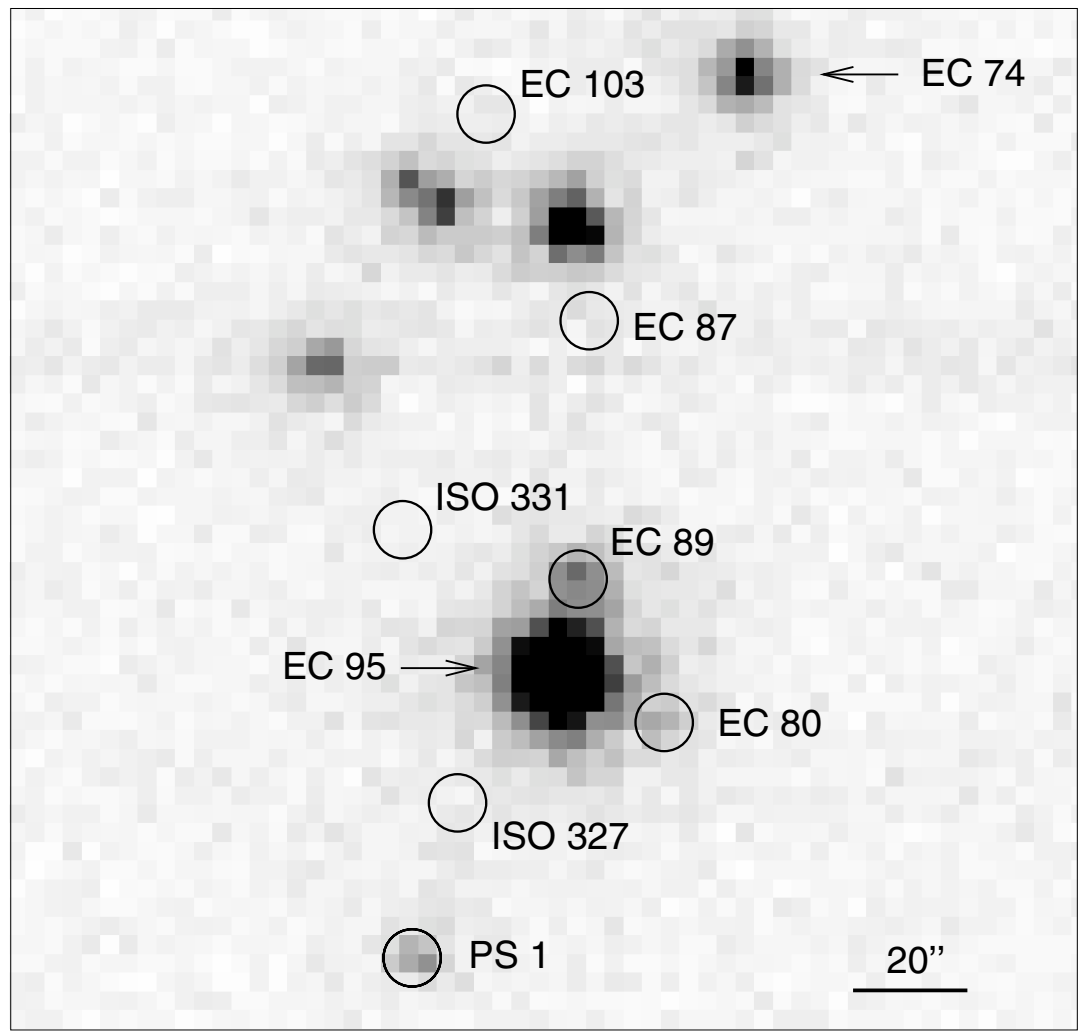

Fig. 1. The central $\sim 3^{\prime} \times 3^{\prime}$ region of the $X M M$-Newton image of the Serpens cloud. The image is the sum of the MOS $1+\operatorname{MOS} 2+\mathrm{PN}$ exposures of the three merged data sets (summed exposure time of $46.1 \mathrm{ks}$ for the PN camera and $52.4 \mathrm{ks}$ for each of the two MOS cameras) in the [0.2-10] keV energy band, binned to $3.25^{\prime \prime} \times 3.25^{\prime \prime}$ pixels, and is displayed with a linear intensity scale (white $=0$ counts, black $=100$ counts). The infrared positions of the class I protostars in this area (Kaas et al. 2004) are marked by circles and labeled.

Furthermore, we searched for strongly variable X-ray sources by comparison of the three observations. On timescales from months to years, variations by factors of $\sim 2-4$ are very common among YSOs and coronally active stars in general (e.g. Stauffer et al. 1994; Marino et al. 2003), and therefore we focused on objects that exceed this usual level of variability. The only remarkable object in this context is the class II object EC 74, which appears much brighter in the merged X-ray data set than in Obs1. The X-ray properties of EC 74 will also be discussed below in detail.

For all sources mentioned above we used SAS to extract lightcurves in circular apertures and in the [0.2-10] keV energy band. We used aperture radii of 6" for EC 80 and EC 89, which are very close to the very bright source EC 95, and 12.5" for PS 1, EC 53, and EC 74. The background was extracted from a circle with $30^{\prime \prime}$ radius in a source-free region. The background-subtracted lightcurves are shown in Fig. 2. EC 74, EC 89, and PS 1 display strong variability, which will be discussed below.

We performed a spectral analysis for those objects that have at least 100 source counts in one observation, which we consider as a minimum for a meaningful spectral analysis. This is the case for EC 74, EC 89, and PS 1 during Obs2. Pulse hight spectra for these sources and suitable background regions were extracted from the data of Obs2. Since EC 89 is affected by the PSF wings of the very bright source EC 95, we used a background region south-west of EC 95 at the same radial distance from EC 95 as the source region. We built the corresponding redistribution matrix files and ancillary response files appropriate for the position and size of the source extraction region, grouped the spectra with 25 counts
Table 2. Results of the fits to the X-ray spectra. The table gives the best fit values for the hydrogen column density $N_{\mathrm{H}}$ and the plasma temperature $T_{\mathrm{X}}$ with the associated $1 \sigma$ uncertainties, the intrinsic extinction corrected X-ray luminosity integrated over the [0.2-10] keV band, and the fit statistic $\chi^{2} / \nu$.

\begin{tabular}{cccccc}
\hline \hline Object & Obs & $\begin{array}{c}N_{\mathrm{H}} \\
{\left[10^{22} \mathrm{~cm}^{-2}\right]}\end{array}$ & $\begin{array}{c}T_{\mathrm{X}} \\
{[\mathrm{MK}]}\end{array}$ & $\begin{array}{c}L_{\mathrm{X}} \\
{[\mathrm{erg} / \mathrm{s}]}\end{array}$ & $\chi^{2} / v$ \\
\hline EC 74 & Obs2 & $2.3_{-0.2}^{+0.2}$ & $55_{-6}^{+9}$ & $\sim 8 \times 10^{30}$ & 0.76 \\
EC 89 & Obs2 & $3.3_{-1.3}^{+6.7}$ & $20_{-4}^{+10}$ & $\sim 2 \times 10^{30}$ & 0.16 \\
PS 1 & Obs2 & $7.9_{-0.7}^{+0.8}$ & $23_{-2}^{+2}$ & $\sim 12 \times 10^{30}$ & 1.18 \\
\hline
\end{tabular}

per bin (5 counts per bin for EC 89), and performed spectral fits with the Sherpa package contained in CIAO. We used the XSPEC model "mekal" describing the emission from a thermal plasma spectrum and "wabs" for the absorption model. The fitting results are described below and summarized in Table 2. During Obs1 and Obs3, none of these sources yielded more than 100 counts, and therefore a meaningful spectral analysis of the sources in these observations was not feasible.

\section{Properties of the individual sources}

\section{1. $P S 1(=H B$ 1)}

The object PS 1 was identified as an IRAS source by Hurt \& Barsony (1996) and is sometimes referred to as HB 1. Kaas (1999) identified a faint $(K=14.37)$ near-infrared counterpart for this object, and Kaas et al. (2004) found it to be a class I protostar according to its infrared spectral energy distribution. 



Fig. 2. XMM-Newton-EPIC-PN lightcurves of the class I protostars PS 1, EC 53, EC 80, and EC 89, the class II object EC 74, and the flat spectrum source EC 95. The histograms show the number of counts per 20 min time bin after correction for background. In the plot for EC 95, the asterisks show the counts in the background region, scaled to the area of each source extraction region.

Haisch et al. (2004) recently resolved the source into a binary with a separation of $1.46^{\prime \prime}$; since this is clearly well below the resolution limit of the XMM-Newton data, we cannot decide which of the infrared components is the X-ray source. As the mid-infrared emission of the system observed by ISO is also unresolved, it is not clear whether both or only one of the components are class I protostars. However, since the two components display very similar $K-L$ colors (Haisch et al. 2004), we assume here that both are class I protostars.

Our XMM-Newton lightcurve (see Fig. 2) shows strong temporal variability for PS 1 . In Obs1 we see a decreasing count rate over the first $\sim 2 \mathrm{~h}$ of the observation; this may be 

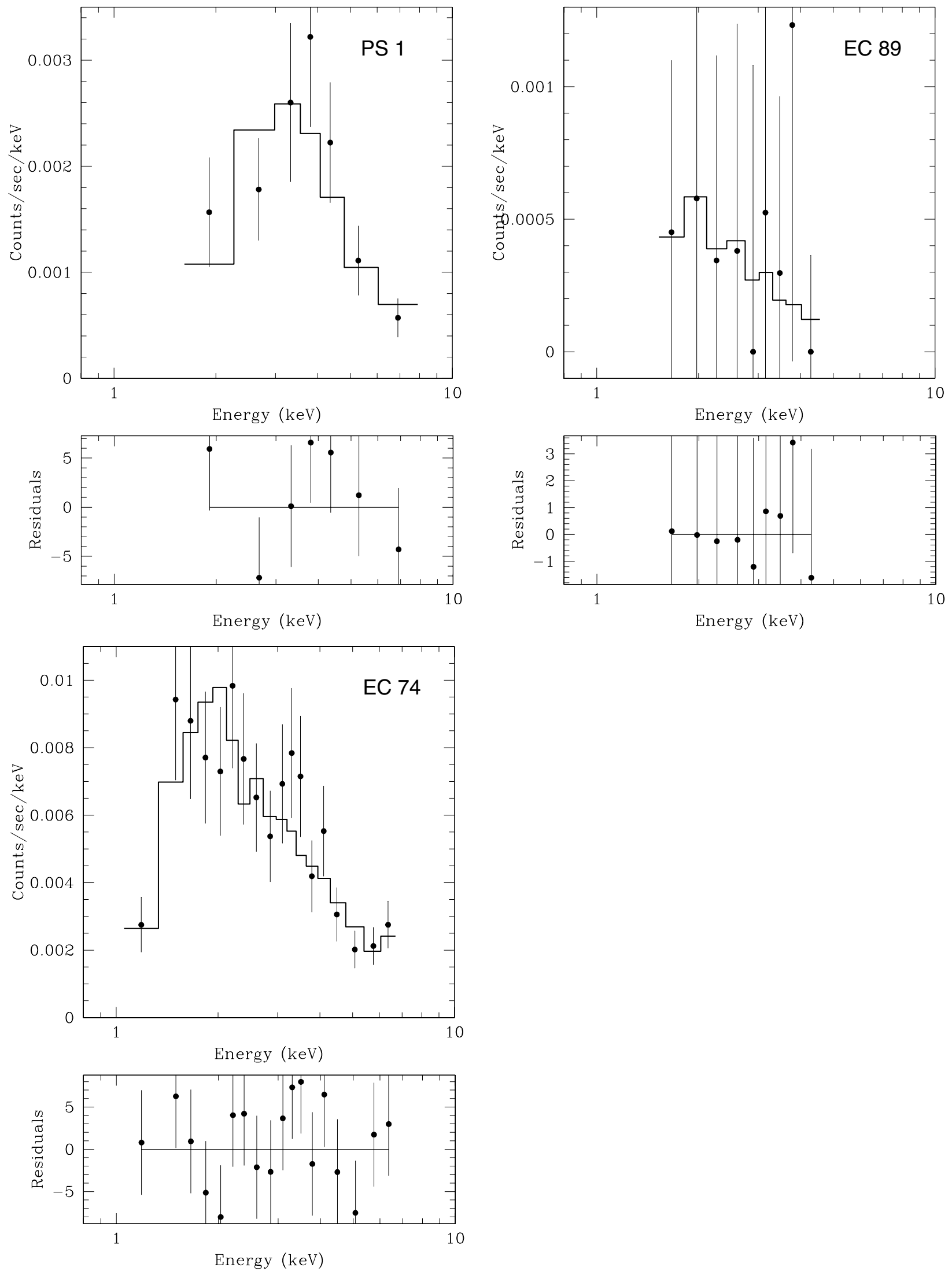

Fig. 3. XMM-Newton-EPIC-PN spectra of the class I protostars PS 1 and EC 89, and the class II object EC 74. The solid dots with error bars show the observed spectrum, the histogram shows the best fit model. 
the late decay phase of a flare that occurred before the beginning of the observation. During Obs2, the lightcurve starts at a much higher value and shows a decay over about $2 \mathrm{~h}$; this suggests a rather strong flare that started before the beginning of the Obs2. During Obs3 the lightcurve shows no significant variability and the mean count rate is roughly consistent with the levels observed at the end of Obs1 and Obs2, i.e. after the flares during that observations. The fit to the X-ray spectrum extracted from Obs 2 yields a plasma temperature of $23 \mathrm{MK}$ and a hydrogen column density of $8 \times 10^{22} \mathrm{~cm}^{-2}$; using the canonical relation $N_{\mathrm{H}} \sim 2 \times 10^{21} \mathrm{~cm}^{-2} \times A_{\mathrm{V}}$ [mag] (see Predehl \& Schmitt 1995; Ryter 1996), this hydrogen column density corresponds to an extinction of $A_{\mathrm{V}} \sim 40 \mathrm{mag}$. The spectral fit was also used to compute the intrinsic (extinction-corrected) $\mathrm{X}$-ray luminosity by integrating the model source flux over the $[0.2-10] \mathrm{keV}$ band. The X-ray luminosity derived from the spectral fit is $1.2 \times 10^{31} \mathrm{erg} / \mathrm{s}$.

\subsection{EC 53}

EC 53 is a rather bright $(K=10.9)$ class I protostar, and displays a prominent cometary shape in the near-infrared images (see, e.g., Eiroa \& Casali 1992). EC 53 was detected as a very weak source in the data of Obs1. Due to its faintness, the lightcurve (Fig. 2) is quite noisy, making the detection of variability difficult.

While the source is too weak to extract a useful X-ray spectrum, we had estimated an X-ray luminosity of $\sim 2 \times 10^{30} \mathrm{erg} / \mathrm{s}$ from the observed count rate in Obs1 and the extinction estimate of $A_{\mathrm{V}, \mathrm{IR}}=16 \mathrm{mag}$ (Eiroa \& Casali 1992). With the bolometric luminosity estimate of $L_{\mathrm{bol}}=0.9 L_{\odot}$ for EC 89 (Eiroa \& Casali 1992) we derive a fractional X-ray luminosity of $\log \left(L_{\mathrm{X}} / L_{\mathrm{bol}}\right)=-3.3$.

\subsection{EC 80}

EC 80 is a rather faint $(K=13.6)$ class I protostar. In our $X M M-N e w t o n$ image it is detected as a very faint source close to the very bright X-ray source EC 95 . Due to the faintness of the source, our XMM-Newton lightcurve (Fig. 2) is quite noisy, and does apparently not show strong variations. Note, however, that due to the low number of detected counts, variations by a factor of $\sim 2-3$ would hardly be detectable.

The faintness of the source also prevented us from extracting a useful X-ray spectrum. Since no extinction estimate is available, we cannot estimate an $\mathrm{X}$-ray luminosity for this object.

\subsection{EC 89}

EC 89 is a relatively bright $(K=11.6)$ class I protostar. Since it is located at a separation of only $19^{\prime \prime}$ from the very bright X-ray source EC 95, the X-ray emission from EC 89 is blended with the extended PSF of EC 95, and was therefore not recognized as an individual source in Obs1.

The lightcurve (Fig. 2) suggests an impulsive increase of the count rate with a very short $(\$ 20 \mathrm{~min})$ rise time and a decay time of $\sim 1$ h during Obs1. A similar event, although with longer rise and decay times is seen during Obs2, while Obs 3 seems to show a slowly rising count rate followed by a sharp decrease. The lightcurve suggests frequent flaring activity of this source. To be cautions, however, we emphasize that the statistical significance of these events is not very high, and some part of the variations may also be due to random fluctuations.

The X-ray spectrum of EC 89 during Obs2 is quite noisy, in part because we had to use a very small extraction region due to the location of EC 89 in the extended PSF wings of the very much brighter source EC 95 . In order to correct the spectrum of EC 89 as far as possible for the contamination by photons from EC 95, we used a background region at the same radial distance from EC 95 as the source region for EC 89. A singletemperature plasma plus absorption model yielded a statistically acceptable fit for a plasma temperature of $T_{\mathrm{X}} \sim 20 \mathrm{MK}$ and a hydrogen column density of $N_{\mathrm{H}} \sim 3.3 \times 10^{22} \mathrm{~cm}^{-2}$. Due to the low number of counts in the spectrum, these fitting parameters are associated with quite large uncertainties. Furthermore, we found that the fit solution also depends on the bin size used to group the spectrum; the use of larger spectral bins resulted in higher fit values for $N_{\mathrm{H}}$ and lower values for $T_{\mathrm{X}}$. We prefer the bin size and the fit solution given above because in this case the hydrogen column density derived from the X-ray spectral fit agrees quite well with the extinction estimate based on the near-infrared colors of $A_{\mathrm{V}, \mathrm{IR}}=17 \mathrm{mag}$ (Eiroa \& Casali 1992) using the canonical relation $N_{\mathrm{H}} \sim 2 \times 10^{21} \mathrm{~cm}^{-2} \times A_{\mathrm{V}}[\mathrm{mag}]$ (see Predehl \& Schmitt 1995; Ryter 1996).

The X-ray luminosity derived from the spectral fit parameters is $L_{X}=2 \times 10^{30} \mathrm{erg} / \mathrm{s}$. Note that this is the average value during Obs2, including the flare. With the bolometric luminosity estimate of $L_{\mathrm{bol}}=0.61 L_{\odot}$ for EC 89 (Eiroa \& Casali 1992) we derive a fractional X-ray luminosity of $\log \left(L_{\mathrm{X}} / L_{\text {bol }}\right)=-3.1$.

\subsection{EC 74}

EC 74 is a class II object, i.e. a T Tauri star with circumstellar material. Its X-ray lightcurve (Fig. 2) displays strong variability: the source is very weak during Obs1, much $(\sim 10 \times)$ brighter during Obs2, and again faint in Obs3. The shape of the lightcurve during Obs2 does not reflect the shape of a "typical" impulsive flare, in which the countrate usually rises quickly, typically within $\lesssim 1 \mathrm{~h}$, followed by an slow, exponential decay to the pre-flare level. Instead, the lightcurve of EC 74 seems to suggest a slowly increasing count rate during the first half of Obs2, that may be followed by a slow decline; the statistical significance of these variations is, however, rather weak, and the Obs2 lightcurve is also consistent with the assumption of a constant count rate. The temporal behavior of the X-ray emission from EC 74 may well be explained by rotational variability. If, for example, the X-ray emission is dominated by one or a few relatively small active coronal regions, large differences of the observed X-ray luminosities are found between times when this active region is on the back side of the star and when it is on the front side. Another possibility would be a long duration, or "gradual" flare during Obs2 (see for 
example Grosso et al. (2004) for such an event on the $\mathrm{T}$ Tauri star $\mathrm{LkH} \alpha 312$ in M 78). Furthermore, a combination of a cooling flare plus rotational modulation (cf. Stelzer et al. 1999) would also be able to explain the shape of the observed lightcurve.

The spectrum of EC 74 can be well fitted by a model with plasma temperature of $55 \mathrm{MK}$ and a hydrogen column density of $2.3 \times 10^{22} \mathrm{~cm}^{-2}$. This hydrogen column density corresponds to an extinction of $A_{\mathrm{V}} \sim 11 \mathrm{mag}$, which agrees well with the extinction derived from near-infrared data of $A_{\mathrm{V}, \mathrm{IR}} \sim 13 \mathrm{mag}$ (Eiroa \& Casali 1992). The X-ray luminosity derived from the spectral fit is $8.3 \times 10^{30} \mathrm{erg} / \mathrm{s}$. The corresponding fractional $\mathrm{X}$-ray luminosity is $\log \left(L_{\mathrm{X}} / L_{\mathrm{bol}}\right)=-2.7$. Note that these values refer to the "high activity stage" during Obs2; in Obs1 and Obs3 the X-ray luminosity is about 10 times smaller.

\subsection{EC 95}

Finally, we briefly mention the lightcurve of the very strong $\mathrm{X}$-ray source EC 95, which is a flat-spectrum object. The X-ray properties of this object (based on the data of Obs1) were discussed in detail by Preibisch (2003), and this will not be repeated here. The lightcurve shows the general trend of a declining count rate, but no significant flares can be seen.

\section{Discussion}

To connect our results to the general knowledge of the X-ray properties of protostars, we consider a recent X-ray study of the $\rho$ Oph cloud, which also harbors a significant population of class I protostars. Imanishi et al. (2001) performed a $100 \mathrm{ks}$ Chandra ACIS observation of $\rho$ Oph and it is worthwhile to summarize their results on the class I protostars here for comparative purposes. They detect X-ray emission from 7 of the 10 class I protostars in their observed region, derive X-ray luminosities between $1 \times 10^{29} \mathrm{erg} / \mathrm{s}$ and $3 \times 10^{30} \mathrm{erg} / \mathrm{s}$, and plasma temperatures in the range 20-100 MK. They find that the class I protostars exhibit stronger temporal variability, i.e. seem to flare more frequently, than the more evolved class II+III objects in $\rho$ Oph. They also conclude that the ratio of the X-ray luminosities in the flaring and quiescent state is higher in the class I protostars than in class II+III objects.

\subsection{Detection fraction and $X$-ray luminosities}

In our XMM-Newton study of the Serpens cloud we detect four out of 19 known class I protostars in the region, i.e. $21 \%$. This detection fraction $^{2}$ is considerably lower than the $70 \%$ in the Chandra study of $\rho$ Oph. One reason for this difference is that the $\rho$ Oph cloud is about two times closer than the Serpens

\footnotetext{
${ }^{2}$ It is interesting to note that the detection fractions among the more evolved YSOs in Serpens is even lower: just two (i.e. 15\%) of the 13 flat spectrum objects from Kaas et al. (2004) and only four (i.e. 9\%) of the 43 class II T Tauri stars listed by Kaas et al. (2004) are detected in X-rays. One reason for this relatively low X-ray detection fractions is that the sample of cluster members was identified in very deep infrared observations.
}

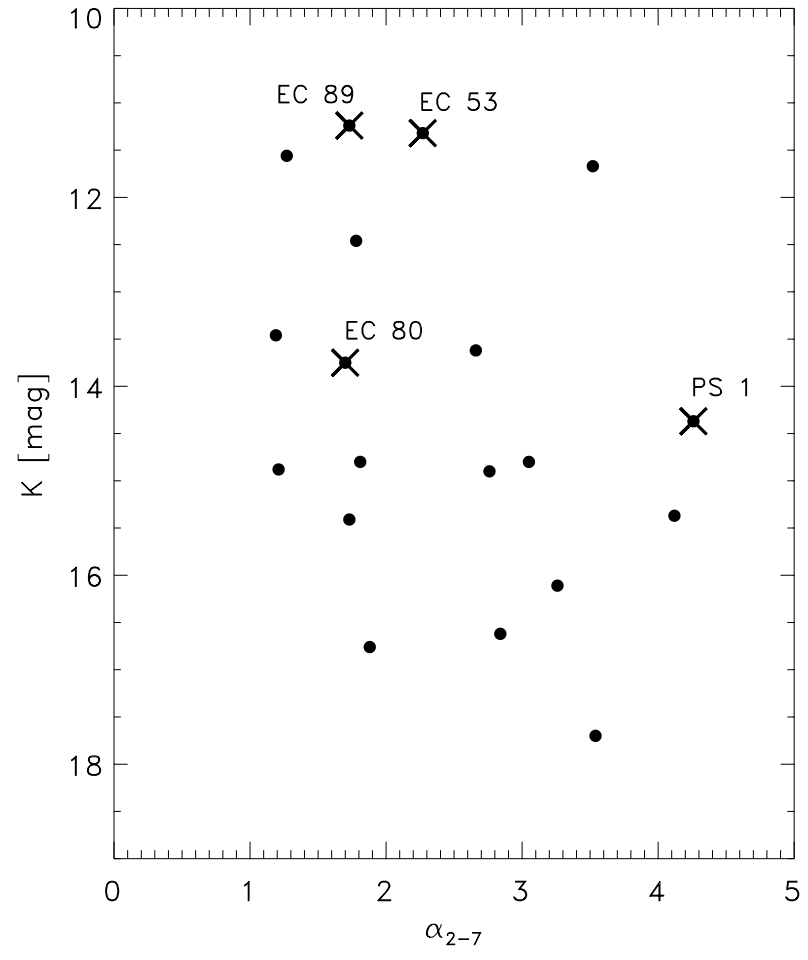

Fig. 4. $K$-band magnitude versus spectral index between $2.2 \mu \mathrm{m}$ and $6.7 \mu \mathrm{m}, \alpha_{2-7}$ for the class I protostars in Serpens from the study of Kaas et al. (2004). The four X-ray detected objects are marked by $\mathrm{X}$ signs and labeled.

cloud. Our derived X-ray luminosities of the three class I protostars in Serpens are around and above the top of the $L_{\mathrm{X}}$ distribution found for the $\rho$ Oph protostars.

The detectability of the class I protostars is clearly limited by the very high extinction. As the extinction in the soft X-ray band around $\sim 1 \mathrm{keV}$ is roughly comparable to that in the nearinfrared $K$-band (which is about 10 times lower than in the optical $V$-band), it is interesting to compare the $K$-band magnitudes of the X-ray detected and undetected class I protostars in Serpens. In Fig. 4 we plot the $K$-band magnitudes against the spectral index between $2.2 \mu \mathrm{m}$ and $6.7 \mu \mathrm{m}, \alpha_{2-7}$, for the class I protostars in Serpens listed in Kaas et al. (2004). The four $\mathrm{X}$-ray detected objects are all among the brighter (in $K$-band) part of the infrared sample. This means that the X-ray detected class I protostars are more luminous (in terms of bolometric luminosity) and/or suffer less extinction than the undetected objects. The spectral index $\alpha_{2-7}$ is a measure of how deeply the object is embedded in its circumstellar disk and/or envelope and may be viewed as a tracer of the evolutionary state of the YSO. EC 53, EC 80, and EC 89 are among less deeply embedded objects and therefore may be in a more advanced state of evolution than PS 1, which is the class I protostar with the largest spectral index in the Serpens sample.

\subsection{Flare frequency of the class I protostars}

In our XMM-Newton data we find a tendency that the class I protostars are more variable than the class II+III objects: we find at least four flare-like events in the lightcurves of the four 
class I protostars, in contrast to just two flare-like variations in the lightcurves of the nine X-ray emitting class II and III YSOs (on EC 67 and EC 135). A detailed comparison of the variability properties of the different classes of YSOs in Serpens is very difficult due to the small source numbers and the low count rates and correspondingly high Poisson fluctuations in many sources. However, it is interesting to compare our sample of Serpens class I protostars to a much larger T Tauri star sample: Feigelson et al. (2002) investigated the variability of solar-mass pre-main sequence stars in the Orion Nebula Cluster based on two $\sim 12 \mathrm{~h}$ long Chandra observations; they saw 30 flares on the $43 \mathrm{~T}$ Tauri stars and conclude from this that, on average, any $\mathrm{T}$ Tauri star shows one flare every 1.4 days $(34 \mathrm{~h})$. For our sample of X-ray detected class I protostars in Serpens we estimate a flare frequency of one flare per object every $\sim 13 \mathrm{~h}^{3}$, i.e. more than twice as often as typical for the T Tauri star in Orion. Due to the small number of objects in our protostar sample, this difference in flare rates is of low statistical significance, but nevertheless is a hint that the class I protostars flare more frequently than more evolved YSOs. This result is in good agreement with the conclusions drawn by Imanishi et al. (2001) for the protostars in $\rho$ Oph and the plenty of flares that have been observed on many class I protostars (e.g. Grosso et al. 1997; Koyama et al. 1996, Tsuboi et al. 2000).

\subsection{Implications on the origin of protostellar $X$-ray emission}

It is still unclear whether the X-ray emission from protostars is produced by the same mechanisms as in older $\mathrm{T}$ Tauri stars, i.e. presumably by strongly enhanced solar-like coronal activity, or whether different (non-coronal) emission mechanism may be involved. Most X-ray properties of the class I protostars, the absolute and fractional X-ray luminosities and the plasma temperatures, are very similar to those of T Tauri stars. However, the higher level of flare-like variability in protostellar $\mathrm{X}$-ray emission, which is inferred from several X-ray observations, including our Serpens results, may indicate different origins of the high-energy emission from protostars and $\mathrm{T}$ Tauri stars.

The strong flare-like variability of the X-ray emission from class I protostars supports theoretical models assuming reconnection events in twisted magnetic field lines between the protostar and the circumstellar disk as a source of X-rays. The basic outline of these models is a follows (for details, see, e.g., Hayashi et al. 1996; Montmerle et al. 2000): The protostar and its circumstellar disk are thought to be magnetically coupled. The initially dipolar stellar magnetic field lines anchored to the inner parts of the accretion disk are twisted around and inflated due to the differential rotation between the star of the disk. This causes the buildup of increasingly large toroidal magnetic

\footnotetext{
3 We assume two flares for EC 89 and two flares for PS 1, i.e. four flare-like events seen on four objects observed for $12.8 \mathrm{~h}$. Note that all our X-ray detected class I protostars would also have been detected in the merged data set without their flares; this implies that our flare frequency is not overestimated by inclusion of objects that were only detected due to a flare.
}

fields, until the contact of antiparallel field lines leads to reconnection events. These reconnection processes transform magnetic field energy ultimately into thermal energy and heat the matter in the magnetic loops to very high temperatures of up to $\sim 10^{8} \mathrm{~K}$, causing bursts of $\mathrm{X}$-ray emission when the plasma cools down on timescales of a few hours. The nature of this mechanism is inherently non-continuous and should produce a sequence of (perhaps quasi-periodic) bursts of X-ray emission. In contrast to coronal emission mechanisms, where a significant part of the X-ray flux seems to come from long-lived loops that emit in a more steady (quiescent) fashion, the protostardisk interaction model therefore predicts a higher level of flarelike variability. This is in agreement with the observational results discussed above.

Since the scenario of Montmerle et al. (2000) requires that the protostar is not yet synchronized with its inner disk, it works only for the more massive protostars, because the magnetic breaking is less efficient to lock rapidly a massive protostar with its inner disk. Nevertheless, this model is relevant for the X-ray detected class I protostars, since the current X-ray detection limit allows us to study only the more luminous and therefore more massive class I protostars. Further observational support for the protostar-disk interaction scenario comes from observations of quasi-periodic X-ray flaring in the class I protostar YLW 15 (Montmerle et al. 2000; however, note that this has been questioned by Favata et al. 2001). The magnetospheric interaction models also provide an interesting basis for a unified explanation of not only the accretion process and the X-ray emission, but also the launching of strong winds and jets (e.g. Goodson et al. 1999).

We finally note that a similar magnetic star-disk coupling may also occur in the older $\mathrm{T}$ Tauri stars (see e.g. Küker et al. 2003). However, the accretion rates in T Tauri systems are much (orders of magnitudes) lower than in protostars, and therefore reconnection events in star-disk fields should provide only a minor fraction of the total $\mathrm{T}$ Tauri X-ray emission, which is dominated by coronal X-rays. Furthermore, it is now well established that accreting $\mathrm{T}$ Tauri stars having 2-3 times lower X-ray luminosities than non-accreting T Tauri stars (e.g. Flaccomio et al. 2003), demonstrating again that the $\mathrm{X}$-ray emission from $\mathrm{T}$ Tauri stars cannot be dominated by magnetic star-disk coupling.

\section{Summary and conclusions}

We have analyzed a set of three XMM-Newton observation of the Serpens dark cloud and detected X-ray emission from 4 of the 19 known class I protostars in this region. We see four flare-like events in the lightcurves of two of the class I protostars, while in the other two objects detection of variability is hampered by the low count rate. The observed high degree of flare-like variability in the X-ray emission of the class I protostars in Serpens is in agreement to X-ray observational results of protostars in other regions. This may support models assuming a non-coronal origin of the protostellar X-ray emission in the magnetic coupling between the protostars and their circumstellar disks. 
Acknowledgements. This work is based on observations with $X M M-N e w t o n$, an ESA Science Mission with instruments and contributions directly funded by ESA member states and the USA (NASA).

\section{References}

Eiroa, C., \& Casali, M. M. 1992, A\&A, 262, 468

Favata, F., \& Micela, G. 2003, Space Sci. Rev., 108, 577

Favata, F., Micela, G., \& Reale, F. 2001, A\&A, 375, 485

Feigelson, E. D., \& Montmerle, T. 1999, ARA\&A, 37, 363

Feigelson, E. D., Garmire, G. P., \& Pravdo, S. H. 2002, ApJ, 572, 335

Feigelson, E. D., Gaffney, J. A., Gramire, G., Hillenbrand, L. A., \& Townsley, L. 2003, ApJ, 584, 911

Flaccomio, E., Damiani, F., Micela, G., et al. 2003, ApJ, 582, 398

Giovannetti, P., Caux, E., Nadeau, D., \& Monin, J.-L. 1998, A\&A, 330,990

Glassgold, A. E., Feigelson, E. D., \& Montmerle, T. 2000, in Protostars and Planets IV, ed. V. Mannings, A. P. Boss, \& S. S. Russel (Tucson: University of Arizona Press), 429

Goodson, A. P., Böhm, K.-H., \& Winglee, R. M. 1999, ApJ, 524, 142

Grosso, N., Montmerle, T., Feigelson, E. D., et al. 1997, Nature, 387, 56

Grosso, N., Montmerle, T., Feigelson, E. D., \& Forbes, T. G. 2004, A\&A, 419, 653

Haisch, K. E. Jr., Greene, T. P., Barsony, M., \& Stahler, S. W. 2004, AJ, 127, 1747

Hayashi, M. R., Shibata, K., \& Matsumoto, R. 1996, ApJ, 468, L37

Hurt, R. L., \& Barsony, M. 1996, ApJ, 460, L45

Imanishi, K., Tsujimoto, M., \& Koyama, K. 2001, ApJ, 563, 361
Jansen, F., Lumb, D., Altieri, B., et al. 2001, A\&A, 365, L1

Kaas, A. A. 1999 , AJ, 118, 558

Kaas, A. A., Olofson, G., \& Bontemps, S. 2004, A\&A, 421, 623

Koyama, K., Hamaguchi, K., Ueno, S., Kobayashi, N., \& Feigelson, E. D. 1996, PASJ, 48, L87

Küker, M., Henning, Th., \& Rüdiger, G. 2003, ApJ, 589, 397

Matsumura, S., \& Pudritz, R. E. 2003, ApJ, 598, 645

Marino, A., Micela, G., Peres, G., \& Sciortino, S. 2003, A\&A, 406, 629

Montmerle, T., Grosso, N., Tsuboi, Y., \& Koyama, K. 2000, ApJ, 532, 1097

Predehl, P., \& Schmitt, J. H. M. M. 1995, A\&A, 293, 889

Preibisch, Th. 1998, A\&A, 338, L25

Preibisch, Th. 2003, A\&A, 410, 951

Ryter, C. 1996, Ap\&SS, 236, 285

Sogawa, H., Tamura, M., Gately, I., \& Merrill, K. M. 1997, AJ, 113, 1057

Stauffer, J. R., Caillault, J.-P., Gagne, M., Prosser, C. F., \& Hartmann, L. W. 1994, ApJS, 91, 625

Stelzer, B., Neuhäuser, R., Casanova, S., \& Montmerle, T. 1999, A\&A, 344, 154

Strom, S. E., Vrba, F., \& Strom, K. M. 1976, AJ, 81, 314

Strüder, L., Briel, U., Dennerl, K., et al. 2001, A\&A, 365, L18

Testi, L., \& Sargent, A. I. 1998, ApJ, 508, L91

Tsuboi, Y., Imanishi, K., Koyama, K., Grosso, N., \& Montmerle, T. 2000, ApJ, 532, 1089

Turner, M. J. L., Abbey, A., Arnaud, M., et al. 2001, A\&A, 365, L27

Wilking, B., Bontemps, S., Schuler, R., Greene, T., \& Andre, P. 2001, ApJ, 551, 357 